\title{
False Data Filtering Strategy in Wireless Sensor Network Based on Neighbor Node Monitoring
}

\author{
https://doi.org/10.3991/ijoe.v13i03.6869 \\ Haishan Zhang* \\ North China University of Science and Technology, Tangshan, China \\ 38775617 @qq. com \\ Xinchun Wang \\ North China University of Science and Technology, Tangshan, China \\ 10759697 @qq. com \\ Chenghui Jia \\ North China University of Science and Technology, Tangshan, China \\ 38775617 @qq. com
}

\begin{abstract}
The injection attack of false data is a common attack form in wireless sensor network. This attack form achieves the purpose of consuming limited network resources and severely threatens the safety of wireless sensor network through consistent sending false data. This paper proposes a type of false data filtering strategy based on neighbor node monitoring. The idea of this strategy is to enable each node to store the neighbor node's information within the two-hop range. In the meantime, the data package determines whether the upstream node is original node or data forwarding intermediate node through whether ACK package is remitted by the upstream node to avoid the impersonation of wireless sensor network node by malicious node. The false data package of malicious node will be filtered within one hop. The simulation experiment verifies the filtering performance and anti-capture performance of this strategy, thus guaranteeing the safety of wireless sensor network.
\end{abstract}

Keywords—wireless sensor network; wastewater monitoring system; ZigBee network; PH parameters

\section{$1 \quad$ Introduction}

Wireless sensor network (WSN) is a newly-emerging field which is combination of sensor technology, network communication and information perception. Wireless sensor network is a type of wireless network composed of static or mobile selforganizing and multi-hop sensors, which can monitor, process and transmit the information within the area in real time, proving users with the information needed [1-3]. Wireless sensor network is regarded as one of the most important technologies in the 21 st century, which fuses the logical information world with the objective physical world. It brings revolutionary changes to the technology of information perception, 
acquisition and processing and is widely applied in national defense and military, environmental monitoring, medical and health care, intelligent buildings and space exploration [4].

The structure of wireless sensor network is shown in Figure 1. We can see from Figure 1 that sensors and nodes are densely arranged in the monitoring area and the sensing data is aggregated to the sink node. The interaction with data of task manage node is achieved through the external network (Internet, satellite-net).

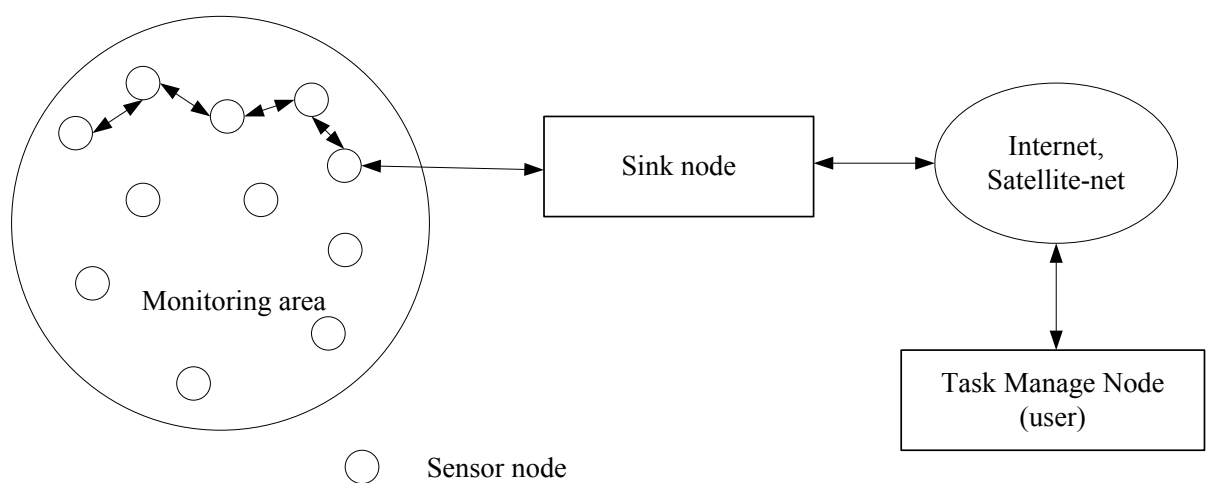

Fig. 1. Structure Diagram of Wireless Sensor Network

We can see from the structure diagram that wireless sensor network nodes and the route in the transmission process are exposed to the outside and thus it is very easy for malicious attackers to capture or forge sensor nodes. Once capturing the node, the confidential information will be stolen and this node will be reformed to malicious node [5]. To attack wireless sensor network, attacks will constantly inject false data through the malicious node, thus occupying network bandwidth and increasing communication overhead. On the one hand, the request by normal nodes will not be responded; on the other hand, the limited power of nodes will be wasted by false data, resulting in the paralysis of wireless sensor network. Therefore, the filtering technology of false data has attracted more and more attention [6].

Some scholars first proposed SEF strategy to tackle the filtering of false data in wireless sensor network. The basic idea is: in the deployment phase of network nodes, each node selects k key storages in the partitions of key pool. Each node is authenticated through the Message Authentication Code (MAC) with pre-stored perception information [7]. After receiving the data package, the node will check the key index corresponded to the MAC. Then, we use this key to verify the validity. If it is illegal, then we can judge that this is false data package and this data package will be discarded. With the proposal of gradual cross-certification, key distribution scheme LBRS based on geographical location information and one-way hash chain authentication, we have made some process in terms of data filtering. However, it is not capable of defending against malicious nodes [8-10]. The fundamental cause why it is very difficult for above strategies to defend against malicious nodes to fabricate false information is that the node receiving data package cannot identify the source of this 
data package. That is to say, the node cannot identify whether upstream nodes are generated or forwarded. Applying false data filtering strategy based on neighbor node monitoring, this paper can identify the source of this data package through ACK package. Thus, it can guarantee that the captured nodes cannot forge the false information in other regions and the false data package will be filtered within one hop in most cases.

\section{False Data Filtering Strategy in WSN Based on Neighbor Node Monitoring}

\subsection{Basic Idea of this Strategy}

In traditional filtering solutions, if attackers capture $\mathrm{T}$ nodes in different regions in the network, they will forge false data package in any region through storage keys in captured nodes. The downstream nodes cannot distinguish this data package is generated or forwarded by upstream nodes, so they can only verify or forward passively [11]. Therefore, if we can identify that the data package is generated by upstream nodes, and then downstream nodes can know whether the node at further distance is the neighbor of this node or not, thus identifying this data package is forged.

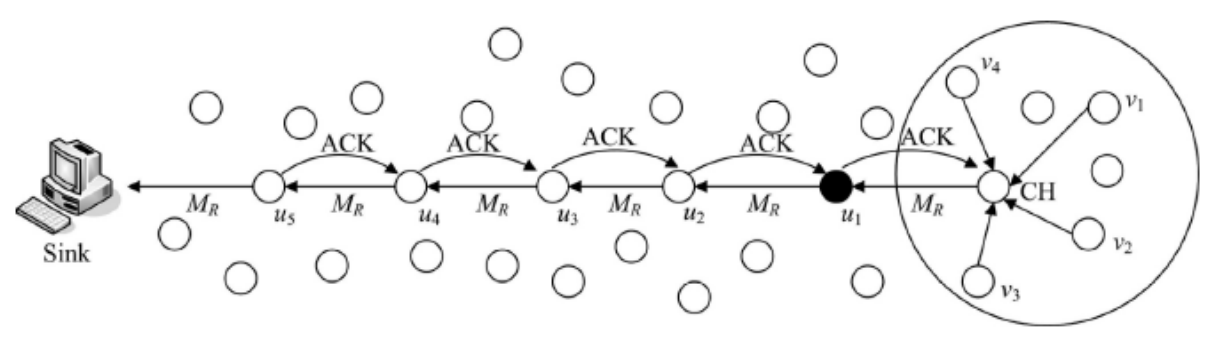

Fig. 2. Schematic Diagram of System Model

Strategy provisions: the neighbor node information within two hops is stored in the node. Each intermediate node, like the cluster-head node, not only needs to forward data package to downstream nodes, but also needs to transmit ACK package to upstream nodes. The model diagram is shown in Figure 2, when a node $u_{2}$ in the network receives the data package $M_{R}$ forwarded by upstream nodes $u_{1}, u_{2}$ forwards ACK package to judge the data package generated is cluster-head node or forwarding node through monitoring. If a node makes the judgement that the node in the last hop is cluster-head node, then it can judge whether each ID in the data package is the neighbor node of the node in the last hop by applying the stored two-hop neighbor node information. If it is not, then it can judge this data package false data package; neighbor nodes can identify malicious nodes use them to generate data package and will automatically send alarming information to downstream nodes [12]. If a node (like $u_{2}$ ) monitors that the ACK package returned by the node $u_{l}$ in the last hop is node $u_{0}$, then 
we can identify that the node $u_{l}$ is the forwarding node. In this case, $u_{l}$ and $u_{0}$ also monitor ACK package and check in the Packet ID in the data package stored buffering area to judge whether there is Packet_ID which is the same as that in ACK package. If there exist, then we can identify the ACK message as legitimate. Or else, we identify the ACK message as abnormal and send abnormal results to node $u_{2}$ in the form of alarm package. When the number of alarm packages in the data package received by node $u_{2}$ exceeds the threshold value $\mathrm{w}$, then we can identify the data package received is false data package. In the transmitting process of alarm package, the real identity of nodes can be guaranteed by one-way chain technology. Sink nodes possess relevant keys of all nodes and small number of escaped false data packages will be detected by nodes after reaching the sink node [13-14].

The format of data package $M_{R}$, ACK package and alarm package is shown in Figure 3. Information like Packet_ID of false data package, its newest one-way chain key and ID of suspicious nodes is contained in the alarm package. Assume $u_{l}$ transmits the false data package to node $u_{2}$ of next hop and their common neighbor $u$ ' judges that it is a false data package applying ACK package. The neighbor node will transmit the data package stored within the two-hop range to $u_{2}$. If $u_{2}$ and $u^{\prime}$ are direct neighbors, the alarm package will be directly transmitted to node $u_{2}$; if $u_{2}$ and $u$ ' are neighbors of two-hop range, then it needs other nodes to forward the alarm package to $u_{2}$ or it will not transmit any data package. After receiving alarm package, $u_{2}$ will use the stored one-way chain key $K_{u}^{i}$, of node $u$ ' to verify the validity of keys in alarm package. If it passes the verification, then $u_{2}$ will count the number of different nodes in alarm package and update the one-way chain keys corresponded to node $u$ '

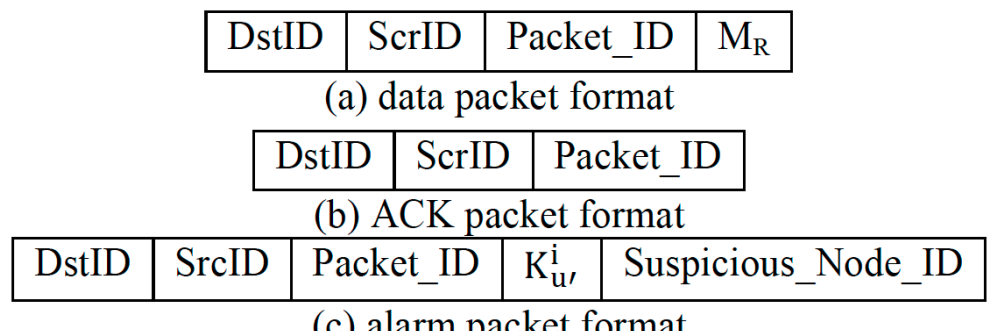

Fig. 3. Various Data Packet Formats

\subsection{Mode of Key Distribution and Initializing Network}

Similar to SEF strategy, we set a public key pool with $\mathrm{N}$ space as $\mathrm{G}$ and $G=\left\{k_{i}: 0 \leq i \leq N-1\right\}$. $\mathrm{i}$ is key index and the key pool is divided into n nonoverlapping key partitions $\left.N_{i}: 0 \leq i \leq n-1\right\}$. Each partition contains $\mathrm{m}$ keys and thus $\mathrm{N}=\mathrm{n} * \mathrm{~m}$. Each key partition can be divided according to $N_{i}=\left\{K_{j} \mid i m \leq j \leq(i+1) m-1\right\}$.

Before the deployment of nodes, each node will store L keys in public key pool randomly and give unique ID showing its identity, a global one-way key generation function $\mathrm{F}(\mathrm{x})$, parameter $\mathrm{ub}, \mathrm{lb}, \mathrm{w}$ and $\mathrm{T}_{\text {wait }} \mathrm{Ub}$ and $\mathrm{lb}$ represent the credibility 
threshold of nodes; w represents the quantity threshold of alarm packages needed in the authentication of certain node; $\mathrm{T}_{\text {wait }}$ represents the time $\mathrm{u}_{1}$ needed to wait for message monitoring after receiving the data package.

After the deployment of nodes in the monitoring area, each node uses random number $R_{u}$ and node ID to generate keys $K_{u}^{m}=F\left(R_{u}, I D_{u}\right)$ and then uses function $K_{u}^{j}=F\left(K_{u}^{j+1}\right)$ to generate $\mathrm{m}+1$ one-way chain keys $<K_{u}^{0}, K_{u}^{1}, \ldots, K_{u}^{m},>$ corresponded to each node. Each node stores $\left[\mathrm{m} / \mathrm{d}_{1}\right]+\mathrm{d}_{2}$ one-way chain keys calculated through its own ID. [m/d1] represents several keys $K_{u}^{d 1}, K_{u}^{2 d 1}, \ldots, K_{u}^{k d 1}, \ldots, K_{u}^{m}$ with the interval of $\mathrm{d}_{1}$ and $\mathrm{d}_{2}$ one-way chain keys to be used are stored, which are initialized as $K_{u}^{0}, K_{u}^{1}, \ldots, K_{u}^{d 2-2}, \ldots, K_{u}^{d 2-1}$. After the usage of first group of keys, the second group of keys are generated and stored into $\mathrm{d}_{2}$ unit by applying neighboring one-way chain keys. Similarly, each node contains its own $I D_{u}$ and the data package of $K_{u}^{0}$ message. After receiving, the neighboring node will record the $I D_{u}$ and $K_{u}^{0}$ of its neighboring node and set the credibility of each neighboring node as 1 . And then, it will send the $I D_{u}$ and $K_{u}^{0}$ of its neighboring node to other neighboring nodes for storage. In this way, each node in the network not only records the $I D_{u}, K_{u}^{0}$ and credibility of its neighboring node, but records the $I D_{u}$ and $K_{u}^{0}$ of its neighboring node within two-hop range.

\subsection{Generation of Data Package}

When the data information needed by users is generated, several sensor nodes around the information will sense this information and transmit this data information to neighboring nodes. When a node receives the data transmitted by more than $T$ different key group nodes, this node informs its neighboring nodes that it is a cluster header node and forms fusion data package $\mathrm{M}$ according to corresponding fusion policies. The data package $\mathrm{M}$ is transmitted to each node in the cluster and they judge the conformity between this data and the data detected based on the application requirement of users and the precision of sensors. If the conformity is good, then randomly select a key $K_{i}$ to generate $M_{i}$ and send it to cluster head node. $M_{i}=\operatorname{MAC}\left(K_{i}\right.$, $\left.M|| I D_{v} \| i\right), \|$ represents the successful connection of data and MAC represents the authentication code of data generated by applying key $K_{i}$. The detailed process is as follow:

$$
v \rightarrow C H: I D_{v}, i, \operatorname{MAC}\left(K_{i}, M|| I D_{v} \| \mid i\right)
$$

When cluster head nodes receive the data fed back by nodes, they will firstly refer to list of neighbor nodes to judge whether this ID is its neighbor node. If it is not, discard this data package and then select $\mathrm{T}$ data packages $M_{R}$ generated by data from different key partitions. After that, transmit the data package to sink node. Then format of data package $M_{R}$ is:

$$
C H_{j} \stackrel{M_{R}}{\longrightarrow} \operatorname{sink}: M, I D_{C H}, K_{C H}^{j}, I D_{v 1}, \ldots, I D_{v i}, i_{1}, \ldots i_{t}, M_{i 1}, \ldots, M_{i t}
$$




\subsection{Sink Node Verification}

After the halfway filtering algorithm, the data complete halfway screening and then the data will be transmitted to sink nodes. After receibving the data package, sink nodes will first check whether the number of keys, ID and MAC in the data package equals to $T$. If not, then discard this data package; if there exist two key indexes orginated from the same key partition, then discard this data package; finally, because sink node has the ID and corrresponding keys of all nodes, small number of false data escaping the filtering of halfway nodes will be regognized and filtered.

\subsection{Analysis of Specific Attacking Identity Strategy of malicious nodes}

Malicious cluster head nodes applying non-neighboring nodes in the network to forge false data package: When malicious nodes apply non-neighboring nodes to transmit false data to downstream nodes, through ACK package, downstream nodes can judge whether it is false data and discards it by the node ID with the two-hop range; if malicious nodes transmit ACK package to neighboring nodes, the neighboring nodes can identify it is illegal data package if the by comparison of Pcaket_ID shows inconsistent results. Moreover, it will transmit alarm package to downstream nodes. When the number of alarm packages from different nodes reach $\mathrm{w}$, this data package will be identified as false data package.

Malicious cluster head nodes applying neighboring nodes to forge false data package: If malicious nodes are captured by neighboring nodes, there will be two identical ID originated from the node from the same partition when transmitting the data package, which is contradictory. This data package will be filtered by downstream nodes and downstream nodes and downstream nodes will receive the alarm package.

Conspiracy attack by malicious cluster head nodes and captured downstream nodes: Many alarm packages will be forwarded when cluster head nodes and captured nodes transmit false data package to each other. During this time, the neighboring area will receive certain number of alarm packages. In this way, false data packages will be filtered.

The above three identity strategies can filter malicious nodes through one hop. 


\section{Strategy Performance and Analysis of Simulation Experiment}

\subsection{Monitoring Capacity of Neighboring Nodes}

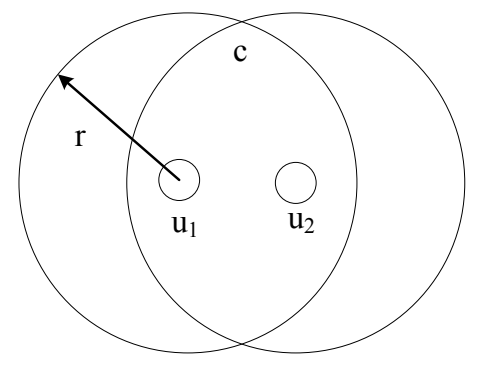

(a)

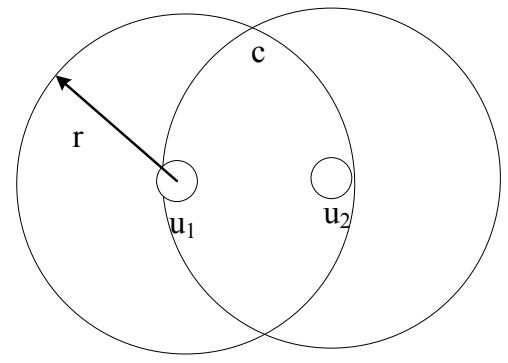

(b)

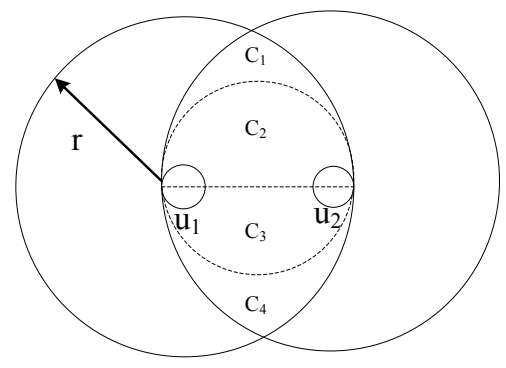

(c)

Fig. 4. Public Neighboring Area of Nodes

Analysis of the number of public monitoring nodes: In terms of nodes that can directly communicate with each other, the number of public neighboring nodes is assocated with distance. The larger the distance, the fewer the public neighboring nodes, as is shown in (a) and (b) in Figure 4. When the interval between $u_{1}$ and $u_{2}$ is the largest communication distance of nodes, the number of public neighboring nodes is the fewest. Assume that the largest communication distance of nodes is $\mathrm{r}$ and $\mathrm{C}$ area is the public area of nodes $u_{1}$ and $u_{2}$, so the minimal area of $\mathrm{C}$ is:

$$
C=\frac{2}{3} \pi r^{2}-\frac{\sqrt{3}}{2} r^{2}
$$

In Figure (b), the proportion of $\mathrm{C}$ area in node communcation area is $40 \%$. Assume the number of neighboring nodes is 20 and these nodes are distributed evenly, and there will be 7 public neighboring nodes for two nodes. Therefore, the number of public monitoring nodes can be guaranteed even under the premise of minimal public area.

Analysis of the number of alarm packages received by nodes in public area $\mathbf{C}$ : When the distance between nodes is the largest commnictaion distance, the 
overlapping area is divided into $\mathrm{C}_{1}, \mathrm{C}_{2}, \mathrm{C}_{3}$ and $\mathrm{C}_{4}$ shown in Figure (c). when node $\mathrm{u}_{2}$ receives the alarm package transmitted by upstream monitoring nodes, this data package must be transmitted or forwarded by certain node in these 4 areas. When $\mathrm{u}_{2}$ receives the alarm package, certain node in $\mathrm{C}_{2}$ at least receives the alarm package of $\mathrm{u}_{2}$ transmitted or forwarded by other 3 areas. This area occupies $83 \%$ in $\mathrm{C}$ area. Certain node in $\mathrm{C}_{1}$ at least receives the alarm package of $\mathrm{C}_{1}$ and $\mathrm{C}_{2}$, occupying $50 \%$ of the node communication area. When $\mathrm{u}_{2}$ receives $\mathrm{m}$ alarm packages transmitted by upstream monitoring nodes, it can be deduced that $\mathrm{C}_{2}$ and $\mathrm{C}_{3}$ at least receives $0.83 \mathrm{~m}$ alarm packages and $\mathrm{C}_{1}$ and $\mathrm{C}_{4}$ at least receives $0.5 \mathrm{~m}$ alarm packages.

The transmission delay of data package in this startegy is low, which exerts little impact on the decision-making of sink nodes. Meanwhile, there will not be great communication overhead and storage overhead, thus satisfying the requiremnet of users.

\subsection{Simulation Experiment}

The parameter configuration is shown in Table 1. We identify that the attack mode is random and take the mean value of 1000 data as the final result. We obtain the filtering capacity, anti-capture performance and algorithm performance by the comparison between SEF and NWFFS through simulation experiment.

Table 1. Parameter Configuration Table

\begin{tabular}{|l|c|}
\hline \multicolumn{1}{|c|}{ Parameter } & Parameter Value \\
\hline Simulation area & $500 * 500 \mathrm{~m}^{2}$ \\
\hline Number of nodes & 2000 \\
\hline Communication radius & $29.8 \mathrm{~m}$ \\
\hline Sensing radius & $29.8 \mathrm{~m}$ \\
\hline Global key pool size & 400 \\
\hline Number of key partitions & 20 \\
\hline The number of keys node stored & 10 \\
\hline Node trust upper threshold ub & 0.8 \\
\hline Node trust Lower threshold lb & 0.1 \\
\hline Alarm package threshold w & 5 \\
\hline Number of key partitions in data package T & 5 \\
\hline
\end{tabular}

Analysis of Filtering Performance: Figure 5 shows that interms of the average number of hops, the average number of transmission hops of false data package in SEF strategy is significnatly higher than that in NWFFS strategy under two different strategies. Morever, the average number of transmission hops increases with the increase of malicious nodes. In NWFFS strategy, the average number of transmission hops is almost a constant value, which shows that the false data filtering strategy in wireless sensor network based on neighbor node monitoring proposed in this paper can effectively mitigate the harm by the attack of false data. 


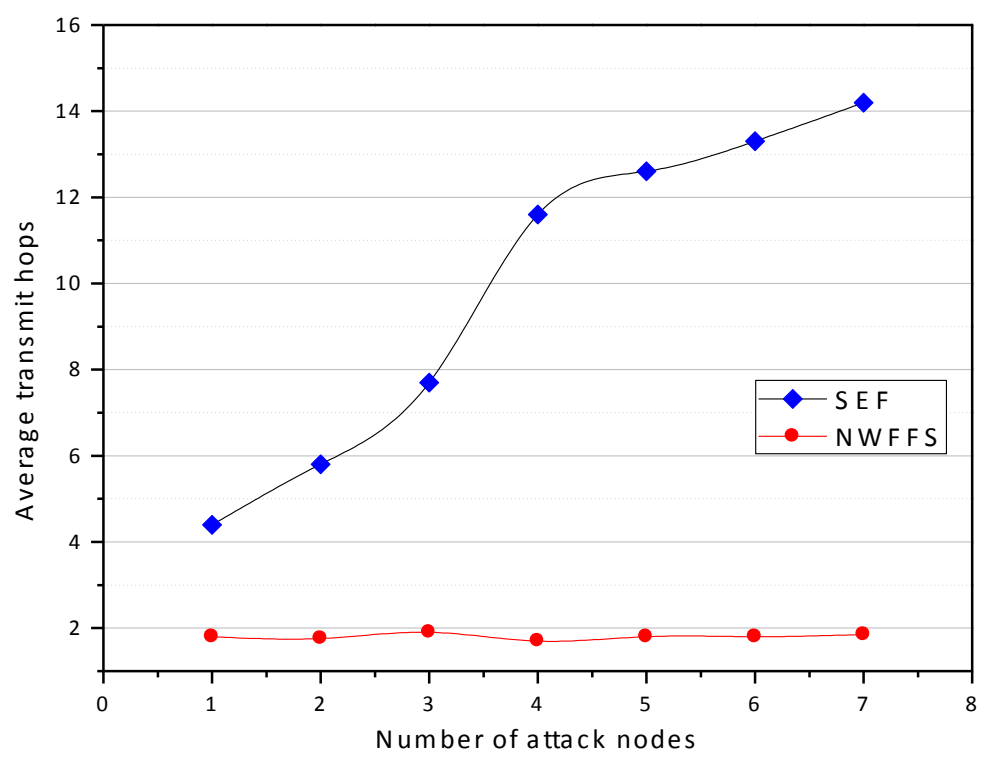

Fig. 5. Average Number of Transmission Hops Before False Data Filtering

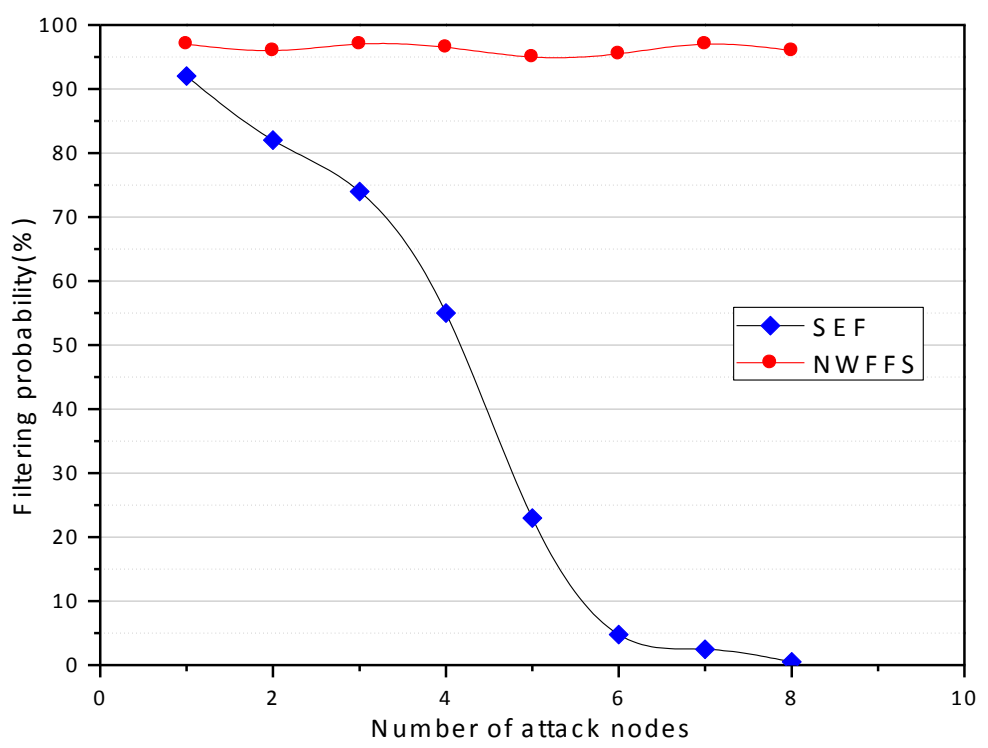

Fig. 6. Filtering Probability of False Data Package

Figure 6 is the comparison between the performance of false data package filtering of two strategies. When the number of captured nodes is relatively less $(<3)$, SEF has good performance. However, with the increase of captured nodes, the filtering capability of SEF declines dramtically. When the number of captured nodes reaches 
8, SEF almost loses its filtering capability. Compared with SEF, NWFFS strategy has almost maintained a good filtering capability of false data package.

Analysis of anti-capture performance: The number of captured nodes is directly proportional to the forgery area of malicious nodes. Therefore, we can use the proportion of area of false data package successfully forged by malicious nodes in total deployement area to measure the anti-capture performance of nodes in this strategy. we can see from Figure 7 that when the number of captured nodes is 5, malicious nodes in SEF strategy can forge 61.3 area events. In NWFFS strategy, malicious nodes can barely forge any area event. When the number of malicious nodes is 49 , malicious nodes in SEF strategy can almost forge any area event while malicious nodes in NWFFS strategy can only forge $3.4 \%$ of area events.

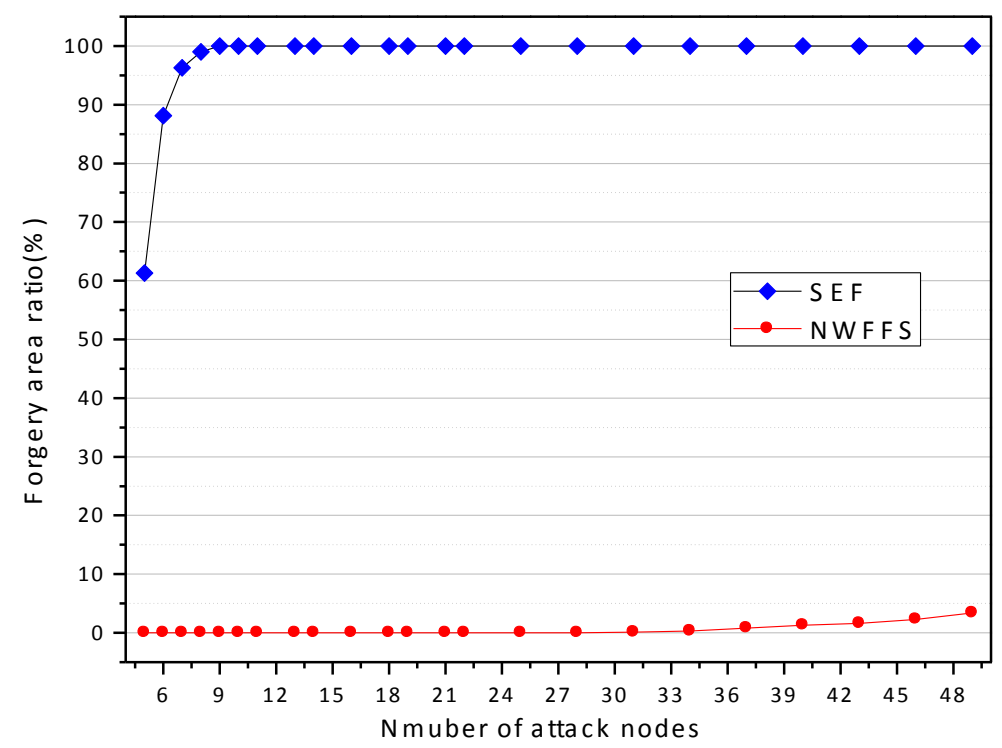

Fig. 7. Proportion of Forgery Area in Total Deployment Area

\section{Conclusion}

This paper proposes a type of false data filtering strategy based on neighbor node monitoring and describes the baisc strategic ideas in detail. After the establishment of the model and analysis of simulation experimental results, we discover that compared with peevious SEF strategy, this strategy has improved significantly in terms of filtering performance and anti-capture capacity, which indicates that this strategy effectively reduces the possibility that malicious nodes apply captured nodes to forge false data. In this way, the impact of malicious nodes on wireless sensor network is reduced and the security of wireless sensor network is further enhanced. 


\section{$5 \quad$ References}

[1] Perrig, A., Stankovic, J., Wagner, D. (2004). Security in wireless sensor networks. Communications of the Acm, 47(6), 53-57. https://doi.org/10.1145/990680.990707

[2] Sandhya, M. K., Murugan, K., Devaraj, P. (2015). Selection of aggregator nodes and elimination of false data in wireless sensor networks. Wireless Networks, 21(4), 1327-1341. https://doi.org/10.1007/s11276-014-0859-y

[3] Lee, H. Y., Cho, T. H. (2009). Detection of false data injection attacks in wireless sensor networks. Nature, 458(7238), 83-91.

[4] Wang, H., Li, Q. (2010). Achieving robust message authentication in sensor networks: a public-key based approach. Wireless Networks, 16(4), 999-1009. https://doi.org/10.1007/ s11276-009-0184-z

[5] Banerjee, T., Veeravalli, V. V. (2015). Data-efficient quickest change detection in sensor networks. IEEE Transactions on Signal Processing, 63(14), 3727-3735. https://doi.org/10.1109/TSP.2015.2432737

[6] Yu, Z., Guan, Y. (2010). A dynamic en-route filtering scheme for data reporting in wireless sensor networks. IEEE/ACM Transactions on Networking, 18(1), 150-163. https://doi.org/10.1109/TNET.2009.2026901

[7] Wang, J., Liu, Z., Zhang, S., Zhang, X. (2014). Defending collaborative false data injection attacks in wireless sensor networks. Information Sciences, 254(1), 39-53. https://doi.org/10.1016/j.ins.2013.08.019

[8] Lin, C., Wu, G., Yu, C. W., Yao, L. (2015). Maximizing destructiveness of node capture attack in wireless sensor networks. The Journal of Supercomputing, 71(8), 3181-3212. https://doi.org/10.1007/s11227-015-1435-7

[9] Chen, Y., Yang, J., Trappe, W., Martin, R. P. (2010). Detecting and localizing identitybased attacks in wireless and sensor networks. IEEE Transactions on Vehicular Technology, 59(5), 2418-2434. https://doi.org/10.1109/TVT.2010.2044904

[10] SUNDAY. (2012). Data dissemination and filtering in wireless sensor network. Journal of the Acoustical Society of America, 113(4), 2217-2217.

[11] Jia, Q. S., Shi, L., Mo, Y., Sinopoli, B. (2012). On optimal partial broadcasting of wireless sensor networks for kalman filtering. IEEE Transactions on Automatic Control, 57(3), 715-721. https://doi.org/10.1109/TAC.2011.2164739

[12] Cheng, P., Qi, Y., Xin, K., Chen, J. (2015). Energy-efficient data forwarding for state estimation in multi-hop wireless sensor networks. IEEE Transactions on Automatic Control, 61(5), 1-1.

[13] Jie, C., Li, J., Yang, S., Fang, D. (2016). Weighted optimization-based distributed kalman filter for nonlinear target tracking in collaborative sensor networks. IEEE Transactions on Cybernetics, 1-14.

[14] Oka, A., Lampe, L. (2008). Energy efficient distributed filtering with wireless sensor networks. IEEE Transactions on Signal Processing, 56(5), 2062-2075. https://doi.org/10.1109/TSP.2007.911496

\section{Authors}

Haishan Zhang was born in Hebei, China, in 1981. He works in the Modern Technology and Education Centre, North China University of Science and Technology, Tangshan 063009, China, and received the Master's degree in 2011 from North 
China University of Science and Technology. His interesting filed is wireless sensor networks, image processing(38775617@qq.com).

Xinchun Wang was born in Hebei, China, in 1977. She works at College of Science, North China University of Science and Technology, Tangshan 063009, China, and received the Master's degree in 2010 from North China University of Science and Technology. Her interesting filed is operation and optimization(10759697@qq.com).

Chenghui Jia was born in Hebei, China, in 1980. He works in the Modern Technology and Education Centre, North China University of Science and Technology, Tangshan 063009, China, and received the Master's degree in 2013 from Nanyang Technological University. His interesting filed is false data filtering,node detection algorithm(38775617@qq.com).

Article submitted 10 February 2017. Published as resubmitted by the authors 23 March 2017. 